ELOHI ELOHI

Peuples indigènes et environnement

1 | 2012

Peuples indigènes et environnement

\title{
El encuentro de Pachamama con la economía global
}

Una reflexión sobre las paradojas del proyecto político boliviano

Jana Jetmarová

\section{(2) OpenEdition}

Journals

Edición electrónica

URL: http://journals.openedition.org/elohi/235

DOI: 10.4000/elohi.235

ISSN: 2268-5243

Editor

Presses universitaires de Bordeaux

Edición impresa

Fecha de publicación: 1 enero 2012

Paginación: 87-102

ISBN: 978-2-86781-842-4

ISSN: 2431-8175

Referencia electrónica

Jana Jetmarová, «El encuentro de Pachamama con la economía global », ELOHI [En línea], 1 | 2012,

Publicado el 01 enero 2013, consultado el 19 abril 2019. URL : http://journals.openedition.org/

elohi/235; DOI : 10.4000/elohi.235 


\section{El encuentro de Pachamama}

\section{con la economía global:}

una reflexión sobre las paradojas del proyecto político boliviano

\section{JANA JETMAROVÁ*}

En los últimos años, en la escena internacional, Bolivia y sobre todo su presidente Evo Morales se han vuelto símbolos del ambientalismo indígena. Bolivia promovió el reconocimiento oficial del Día Internacional de la Madre Tierra de parte de la $\mathrm{ONU}^{1}$. En abril 2010, convocó la primera Conferencia Mundial de los Pueblos sobre el Cambio Climático y Derechos de la Madre Tierra en Tiquipaya $^{2}$. Junto con Ecuador ${ }^{3}$, Bolivia reconoció la naturaleza como una persona

* Universidad Pardubice

1. "Resolución Aprobada por la Asamblea General, 63/278. Día Internacional de la Madre Tierra», Naciones Unidas. Accesible en http://www.un.org/es/comun/docs/?symbol=A/RES/63/278, consultado el 20 de octubre 2011.

2. "Conferencia Mundial de los Pueblos sobre el Cambio Climático y Derechos de la Madre Tierra», CMPCC. Accesible en http://cmpcc.org/adhesiones/, consultado el 25 de enero 2012.

3. «Constitución del Ecuadon», Capítulo séptimo: Derechos de la naturaleza, Artículos 71 - 74, Asamblea constituyente. Accesible en http://www.asambleanacional.gov.ec/documentos/Constitucion-2008. pdf, consultado el 24 de enero de 2012. 
jurídica y ratificó la Ley de Derechos de la Madre Tierra ${ }^{4}$. A nivel internacional lucha por la aprobación de la Declaración Universal de Derechos de la Madre Tierra ${ }^{5}$. Parece que en Bolivia, el país con mayor porcentaje de población indígena en Sudamérica ${ }^{6}$, se está realizando un proyecto político excepcional, que intenta conciliar los conceptos occidentales de modernidad y progreso y los paradigmas indígenas basados en el principio de «vivir bien», un principio que supone el equilibrio y reciprocidad en la sociedad y entre la sociedad y su medio natural y que, traducido a la terminología occidental, implica la necesidad de la sobriedad voluntaria y mantenimiento de la vida sustentable (Huanacuni Mamani 33).

A nivel teórico, el proyecto boliviano representa una síntesis armónica de desarrollo económico, justicia social y políticas ambientales. Pero, como veremos más adelante, es un proceso bastante complejo y lleno de contradicciones. El mayor compromiso del gobierno de Morales consiste en el mejoramiento de la situación social de la población, cuya mayoría vive bajo la línea de la pobreza7. La política económica del Estado se concentra en el aumento de ingresos aprovechando la riqueza natural de Bolivia, lo que en la realidad es prácticamente incompatible con la política de protección medioambiental. Los reconocidos Derechos de la Madre Tierra inevitablemente entran en conflicto con los intereses económicos de la sociedad. Además, el gobierno que se declara proindígena, en sus pasos concretos prácticamente viola los derechos de los pueblos indígenas garantizados constitucionalmente ${ }^{8}$.

En este artículo vamos a analizar los conceptos ideológicos clave del proyecto boliviano y los vamos a poner en el contexto de la política económica real del Estado. A base de este análisis trataremos de contestar la pregunta, si en un país que está interrelacionado con la economía global y dependiente de los caprichos del mercado internacional es posible alcanzar el cumplimiento del «vivir bien». ¿Es posible realmente conciliar los conceptos antropocéntricos del modernismo occidental y los principios holísticos y equilibrados indígenas?

4. «Ley de derechos de la Madre Tierra», Gobernabilidad democrática en Bolivia. Accesible en http:// www.gobernabilidad.org.bo/noticias/2-noticias/704-bolivia-promulga-la-ley-de-derechos-de-la-madre-tierra, consultado el 9 de octubre de 2011.

5. «El discurso de Evo Morales realizado el 22 de abril del 2009 ante la Asamblea General de la Naciones Unidas", Ministerio del Medio Ambiente y Agua. Accesible en http://www.mmaya.gob.bo/\#, consultado el 25 de octubre 2011.

6. "Censo de 2001», Instituto Nacional de Estadística. Accesible en http://www.ine.gob.bo/indice/ visualizador.aspx?ah=PC201 13.HTM , consultado el 5 de octubre de 2011.

7. «Tasa de incidencia de la pobreza, sobre la base de la línea de pobreza nacional (\% de la población)», Banco Mundial. Accesible en http://datos.bancomundial.org/pais/bolivia, consultado el 27 de noviembre de 2011.

8. Por ejemplo, en el Chaco boliviano casi la mitad del área asignada para la explotación de petróleo de parte del gobierno la constituyen los territorios guaranís (Chavez 2010). 


\section{Nueva Constitución: las paradojas del «vivir bien»}

En 2009, Bolivia ratificó la Nueva constitución Política (Borzutzky y Zwart 2009), que redefine el concepto y la función de Estado, garantiza un espectro amplio de derechos indígenas y profundiza (por lo menos en forma escrita) la estrategia de protección del medio ambiente. El texto del Preámbulo se puede interpretar como un intento de crear un nuevo mito nacional. Los principios del Estado plurinacional están anclados en un marco cosmogónico en el que las dimensiones humanas y naturales están profundamente interrelacionadas: «En tiempos inmemoriales se erigieron montañas, se desplazaron ríos, se formaron lagos. Nuestra a mazonia, nuestro c haco, nuestro altiplano y nuestros llanos y valles se cubrieron de verdores y flores. Poblamos esta sagrada Madre Tierra con rostros diferentes, y comprendimos desde entonces la pluralidad vigente de todas las cosas y nuestra diversidad como seres y culturas...» ${ }^{9}$. Esta introducción poética abre un documento que aspira a unir las visiones indígenas con los conceptos del Estado, economía y política occidentales. Como "principios ético-morales de la sociedad plural» la Constitución asume los conceptos indígenas de «vivir bien» o llevar «la vida buena o harmoniosa» (suma qamaña, ñandereko, qhapaj ñan) ${ }^{10}$. Son principios directamente relacionados con la vida sostenible: «vivir bien» significa mantener el equilibrio basado en la reciprocidad no solamente en la sociedad, pero también entre la sociedad y su ambiente natural. En el contexto andino, históricamente las comunidades indígenas mantenían un nivel sostenible del aprovechamiento de las fuentes naturales que aseguraba su recuperación y sostenibilidad para las generaciones venideras. En las cosmovisiones andinas, el principio de equilibrio juega un rol central (Ramírez 2005). Con esta lógica, las exageradas ambiciones individuales perjudican toda la sociedad causando el desequilibrio entre sus miembros (Calestani 59) y el progreso ilimitado de la sociedad daña las entidades vivientes del medio natural con lo que rompe el equilibrio cósmico (Huanacuni Mamani 35).

Asumiendo estos principios a nivel constitucional Bolivia se compromete por lo menos formalmente- a imponer políticas de vida sostenible basada en el respeto al medio ambiente, cuya conservación constituye uno de los «...fines y funciones esenciales del Estado» ${ }^{11}$. El Estado boliviano se obliga explícitamente a «...conservar, proteger y aprovechar de manera sustentable los recursos naturales y la biodiversidad, así como mantener el equilibrio del medio ambiente» ${ }^{12}$.

9. Constitución Política del Estado, preámbulo. Véase "Constitución Política del Estado», 2008, Ministerio de Culturas, accesible en http://www.minculturas.gob.bo/mdcb/normativa/cpe.pdf, consultado el 3 de agosto de 2011.

10. Primera Parte, Título I, Capítulo Primero: Modelo de Estado, Artículo 8, Párrafo I.

11. Primera Parte, Título I, Capítulo Segundo: Principios, Valores y Fines del Estado, Artículo 9, Subpárrafo 6.

12. Cuarta Parte, Título II, Capítulo Primero: Disposiciones Generales, Artículo 342. 
El derecho a «un medio ambiente saludable, protegido y equilibrado» ${ }^{13}$ figura como el primero de los derechos sociales y económicos de los bolivianos. Este derecho constitucional «...debe permitir a los individuos y colectividades de las presentes y futuras generaciones, además de otros seres vivos, desarrollarse de manera normal y permanente. ${ }^{14} \mathrm{La}$ inclusión de «otros seres vivos» representa una importante ampliación del concepto de derechos a las entidades no humanas, lo que culmina en la declaración de los Derechos de la Madre Tierra, como veremos más adelante.

La población indígena tiene garantizado constitucionalmente el derecho a «vivir en un medio ambiente sano" y aprovechar adecuadamente los ecosistemas ${ }^{15}$. Las autonomías indígena originario campesinas ${ }^{16}$ pueden participar activamente en el proceso de "conservación de recursos forestales, biodiversidad y medio ambiente ${ }^{17}$ y pueden ejercer "control y monitoreo socioambiental a las actividades hidrocarburíferas y mineras que se desarrollan en su jurisdicción». ${ }^{18}$

Para cumplir con estos principios teóricos, la constitución prohíbe explícitamente las actividades potencialmente amenazantes para el ambiente: se prohíbe la «importación, producción y comercialización de organismos genéticamente modificados» ${ }^{19}$ así como «la fabricación y uso de armas químicas, biológicas y nucleares...y depósito de residuos nucleares y desechos tóxicos» en el territorio $^{20}$. Asimismo, la constitución impone el deber de sanear los daños causados por las actividades productivas o explotadoras ${ }^{21}$.

Por un lado, la Nueva Constitución parece propugnar un sistema complejo de protección ambiental. Por otro lado, en el mismo documento encontramos notables contradicciones. En la realidad, los derechos ambientales planteados en la primera parte de la Constitución son prácticamente negados en la segunda. La prioridad de las políticas económicas del Estado es «la industrialización de los

13. Primera Parte, Título II, Capítulo Quinto: Derechos Sociales y Económicos, Artículo 33.

14. Primera Parte, Título II, Capítulo Quinto: Derechos Sociales y Económicos, Artículo 33.

15. Primera Parte, Título II, Capítulo Cuarto: Derechos de las Naciones y Pueblos Indígena Originario Campesinos, Artículo 30, Párrafo II, Subpárrafo 10.

16. Según el Capítulo Séptimo de la Constitución, las naciones y los pueblos indígena originario campesinos pueden disponer de autonomía territorial en el marco de la cual pueden ejercer autogobierno «de acuerdo a sus normas, instituciones, autoridades y procedimientos... en armonía con la constitución y la ley» (Artículo 290, Párrafo II). Los artículos 289-296 de la Constitución especifican el proceso de la conformación y delimitación territorial de las autonomías indígena originario campesinas, así como su relación con los distritos municipales.

17. Tercera Parte, Título I, Capítulo Octavo: Autonomía Indígena Originario Campesina, Artículo 304, Párrafo III, Subpárrafo 3.

18. Tercera Parte, Título I, Capítulo Octavo: Autonomía Indígena Originario Campesina, Artículo 304, Párrafo III, Subpárrafo 9.

19. Segunda Parte, Título VIII, Capítulo Primero: Relaciones internacionales, Artículo 255, Párrafo II, Subpárrafo 8.

20. Cuarta Parte, Título II, Capítulo Primero: Medio Ambiente: Artículo 344, Párrafo I.

21. Cuarta Parte, Título II, Capítulo Primero: Medio Ambiente: Artículo 347, Párrafo II. 
recursos naturales», con el fin de liberar el país de la dependencia de la exportación de materias primas y asegurar fuentes de empleo e ingreso económico para el pueblo ${ }^{22}$. Aunque la constitución enfatiza que el proceso de industrialización se realice «en el marco de respeto y protección del medio ambiente» e impone a todas las formas de organización económica la protección medioambiental23, ¿es posible llevar a cabo el proceso de industrialización sin perjudicar o alterar el medio ambiente y los ecosistemas locales? Como evidencian las experiencias en los territorios afectados por las actividades explotadoras, el proceso de industrialización suele tener un impacto socio-ecológico devastador (Kimerling 80).

Las fuentes de materias primas estratégicas, sobre todo hidrocarburos, se encuentran en la parte oriental selvática del país. Es la zona de mayor biodiversidad y al mismo tiempo territorio de varias comunidades indígenas, cuyo medio natural es devastado por el proceso de extracción e industrialización de los recursos no renovables. A pesar de la orientación proindígena oficial del gobierno de Morales y el énfasis en los derechos de los grupos indígenas en la Constitución ${ }^{24}$, son principalmente ellos los que siguen sufriendo las consecuencias negativas de la política extractivista del Estado (Bebbington y Humphreys 2011). No es solamente la gran cantidad de desechos tóxicos la que causa daños ambientales irreversibles: la construcción y mantenimiento de la infraestructura junto con la red de transporte abren el camino a nuevos colonos, cuyos intereses económicos fácilmente pueden entrar en conflicto con las comunidades indígenas locales. Especialmente en las zonas aún relativamente aisladas, el impacto socio-ecológico de la política extractivista en la población indígena puede ser totalmente destructivo (Gavaldá 1999).

Formalmente, la Constitución impone el deber de consultar a la población afectada antes de empezar cualquier actividad explotadora de recursos naturales, especialmente con respecto a las eventuales alteraciones ambientales ${ }^{25}$. No obstante, a pesar del reconocimiento constitucional del derecho a la territorialidad de los grupos indígenas ${ }^{26}$, los recursos naturales son propiedad del pueblo boliviano y su administración corresponde al Estado ${ }^{27}$. Las comunidades indígenas tienen derecho a la consulta previa, monitoreo de los impactos socio-ecológicos y a «la participación de los beneficios de la explotación de los

22. Cuarta Parte, Título I, Capítulo Tercero: Políticas Económicas, Artículo 319, Párrafo I, Cuarta Parte, Título I, Capítulo Primero: Disposiciones Generales, Artículo 311, Párrafo II, Subpárrafo 3, Cuarta Parte, Título I, Capítulo Segundo: Función del Estado en la Economía, Artículo 316, Subpárrafo 6.

23. Cuarta Parte, Título I, Capítulo Primero: Disposiciones Generales, Artículo 312, Párrafo III.

24. Primera Parte, Título II, Capítulo Cuarto: Derechos de las Naciones y Pueblos Indígena Originario Campesinos, Artículos 30-32.

25. Cuarta Parte, Título II, Capítulo Segundo: Recursos Naturales, Artículo 352.

26. Primera Parte, Título II, Capítulo Cuarto: Derechos de las Naciones y Pueblos Indígena Originario Campesinos, Artículo 30, Párrafo I.

27. Cuarta Parte, Título II, Capítulo Segundo: Recursos Naturales, Artículo 349, Párrafo I. 
recursos naturales en sus territorios ${ }^{28}$, pero al mismo tiempo están prácticamente privadas de la posibilidad de manejar independientemente los recursos no renovables de las tierras que habitan. Es el Estado quien administra y decide. En la práctica, el deber constitucional de la consulta previa frecuentemente es omitido o se vuelve solamente un acto formal (Bebbington 2009: 18). Los pueblos indígenas tienen garantizados sus derechos en el papel, pero en la realidad siguen subordinados a los intereses económicos del Estado. La estrategia de las políticas económicas del Estado consiste en la creación de excedentes que puedan ser redistribuidos a los sectores sociales vulnerables y así hacer que de esta manera ellos apoyen el proceso de modernización (Bebbington y Humphreys 2011: 137). En la práctica, el valor económico proveniente de un área es distribuido en favor de otra. Así, los pueblos indígenas ${ }^{29}$ sufren las externalidades negativas de «la política productiva industrial y comercial del Estado» ${ }^{30}$, mientras que las zonas con mayor concentración demográfica son las que gozan de sus beneficios (Bebbington y Humphreys 2011: 142).

Como tratamos de demostrar en nuestro breve análisis, en la Nueva Constitución hay una cantidad de contradicciones. En el marco de las políticas económicas del Estado, cuya prioridad es fortalecer «la infraestructura productiva, manufactura e industrial $\aleph^{31}$, es prácticamente imposible cumplir con los compromisos de protección ambiental y propugnar los derechos de los pueblos indígenas. La necesidad de modernización y desarrollo parece contradecir el principio de «vivir bien». Las protestas indígenas que enfrenta el gobierno de Morales, últimamente la marcha contra la construcción de la carretera interestatal en el Territorio Indígena y Parque Nacional Isiboro-Sécure (TIPNIS) ${ }^{32}$, demuestran la conflictividad y la incompatibilidad práctica de estos dos conceptos.

\section{La defensa de la Madre Tierra: la Pachamama como un actor político}

A pesar de los conflictos entre el principio de «vivir bien» y la política económica del Estado que estimula la explotación de recursos naturales, a nivel internacional el gobierno boliviano se pone en el rol del defensor riguroso del

28. Primera Parte, Título II, Capítulo Cuarto: Derechos de las Naciones y Pueblos Indígena Originario Campesinos, Artículo 3, Párrafo II, Subpárrafo 16.

29. En este lugar consideramos principalmente la situación de las comunidades indígenas de la parte oriental del país. Tenemos en cuenta que los habitantes de las zonas urbanas también se pueden identificar como indígenas (como los pobladores de El Alto).

30. Cuarta Parte, Título I, Capítulo Tercero: Políticas Económicas, Artículo 318, párrafo I.

31. Cuarta Parte, Título I, Capítulo Tercero: Políticas Económicas, Artículo 318, Párrafo III.

32. "Por la defensa de la Madre Tierra, tierra y territorio», CIDOB:Conferedación de los pueblos indígenas de Bolivia. Accesible en http://www.cidob-bo.org/index.php?option=com_content\&view=article\&id= 888: presidente-de-cidob-dio-conferencia-de-prensa-en-la-cop-17-en-durban\&catid=82:noticias\&ltemid=2, consultado el 17 de abril 2012. 
medio ambiente. En la retórica de Evo Morales los temas ambientales son muy frecuentes y forman parte de un discurso político más amplio, basado en la crítica aguda del sistema socioeconómico occidental. «Capitalismo es el peor enemigo de la humanidad $»^{33}$ dice Morales y acusa al sistema basado en la premisa del crecimiento económico ilimitado de agotar irreversiblemente los recursos naturales, causar y continuar empeorando el calentamiento global y de esta manera amenazar las condiciones de la vida misma en el planeta ${ }^{34}$.

En abril de 2010, en Tiquipaya (Cochabamba) se celebró la primera Conferencia Mundial de los Pueblos sobre el Cambio Climático y los Derechos de la Madre Tierra, que fue convocada por iniciativa boliviana frente al fracaso de la Cumbre de Cambio Climático en Copenhague en diciembre de 2009. En la conferencia asistieron más que 35000 personas de unos 140 países, la mayoría de origen indígena (Lindisfarne 1). Las discusiones de los 17 grupos de trabajo resultaron en el «Acuerdo de los Pueblos», que enfatiza los puntos clave de la política ambiental, promovida por Bolivia en la escena internacional. Es un documento detallado que intenta propugnar el principio de «vivir bien» a nivel planetario. Considera ilegítimo el acuerdo de Copenhague, rechaza el llamado sistema capitalista y requiere «...forjar un nuevo sistema que restablezca la armonía con la naturaleza y entre los seres humanos». ${ }^{35}$

Según el Acuerdo de Tiquipaya, no es posible enfrentar los cambios climáticos sin cuestionar sus causas estructurales que consisten en el sistema socioeconómico occidental. «Sólo puede haber equilibrio con la naturaleza si hay equidad entre los seres humanos... $\nu^{36}$, por eso es necesario establecer el equilibrio entre los países más contaminadores y los que sufren las consecuencias ambientales del cambio climático mediante el pago de la deuda climática, que los países desarrollados, productores del $75 \%$ de gases de efecto invernadero, destinarían a los países en vías de desarrollo como una compensación por los daños ecológicos sufridos. Es necesario establecer un «Tribunal Internacional de Justicia Climática» en el marco de la ONU para juzgar el incumplimiento de compromisos ambientales de los países. Asimismo, hay que reconocer la condición específica de los migrantes climáticos. Pero principalmente, el Acuerdo pone énfasis en la necesidad de promover un sistema alternativo basado en «...la recuperación, revalorización

33. «La entrevista con Evo Morales en la Conferencia Climática en Copenhagen el 17 de dicimebre del 2009", Democracy Now. Accesible en http://www.democracynow.org/2009/12/17/bolivian_president_ evo_morales_on_climate, consultado el 15 de octubre 2011.

34. "Bolivia's President Urges Development of Economic System Based on 'How to Live Well'», United Nations: Permanent forum on Indigenous Issues. Accesible en http://www.un.org/News/Press/ docs/2008/hr4944.doc.htm, consultado el 18 de abril 2012.

35. "Acuerdo de los Pueblos», Conferencia Mundial de los Pueblos sobre el Cambio Climático y Derechos de la Madre Tierra. Accesible en http://cmpcc.org/acuerdo-de-los-pueblos/, consultado el 20 de octubre 2011.

36. Ibid. 
y fortalecimiento de los conocimientos, sabidurías y prácticas ancestrales de los Pueblos Indígenas $\star^{37}$. En este sentido, es imprescindible reconocer a la Madre Tierra «...como un ser vivo, con el cual tenemos una relación indivisible» ${ }^{38}$.

El reconocimiento de la Madre Tierra como una entidad viviente representa un desvío importante desde el punto de vista antropocéntrico hacia una visión holística, inspirada en las cosmovisiones indígenas. En el contexto cultural andino existe una relación importante entre los seres humanos y el medio ambiente que a nivel sociopolítico forma la base para la construcción de lo que Healey (85) denomina como una «identidad etno-ecológica». El ambiente natural forma parte integral de las comunidades indígenas, el paisaje y los fenómenos naturales son considerados como entidades vivientes dotadas de su propia voluntad e interrelacionadas con la sociedad humana (De Cadena 354). En el contexto quechua y aymara la tierra es venerada como madre, la Pachamama, los cerros y montañas son respetados como seres guardianes relacionados con los antepasados. Los seres humanos deben mantener una relación armoniosa con las entidades del medio natural, el comportamiento inadecuado causa la alteración del equilibrio cósmico cuyas consecuencias se revelan en forma de desastres naturales (De Cadena 252-257). Con la misma lógica, los fenómenos actuales, que son productos de los cambios climáticos globales, como el derretimiento observable de los glaciares andinos, son interpretados como la consecuencia inevitable del comportamiento erróneo de la humanidad. Y según el Acuerdo de Tiquipaya, son precisamente los pueblos indígenas quienes pueden ofrecer a la humanidad una visión alternativa para restablecer la armonía y el equilibrio entre el hombre y la Pachamama.

Siguiendo los requerimientos del Acuerdo, en diciembre de 2010 Bolivia llegó a ser el primer país del mundo que promulgó explícitamente la Ley de Derechos de la Madre Tierra. Junto con Ecuador, Bolivia reconoció el estatus jurídico de la Madre Tierra (o naturaleza en la terminología occidental), que «...adopta el carácter de sujeto colectivo de interés público». ${ }^{39}$ La Madre Tierra es reconocida como «...el sistema viviente dinámico conformado por una comunidad indivisible de todos los sistemas de vida y los seres vivos $»^{40}$, incluyendo en este marco ecosistémico también a la sociedad humana cuyas «....actividades... deben lograr equilibrios dinámicos con los ciclos y procesos inherentes a la Madre Tierra $»^{41}$. En el marco de la Ley, la sociedad es entendida como una parte

37. Ibid.

38. Ibid.

39. "Ley de Derechos de la Madre Tierra, Capítulo II, Artículo 5», Gobernabilidad democrática en Bolivia. Accesible en http://www.gobernabilidad.org.bo/noticias/2-noticias/704-bolivia-promulga-la-ley-dederechos-de-la-madre-tierra, consultado el 9 de octubre de 2011.

40. Capítulo II, Artículo 3.

41. Capítulo I, Artículo 2, Subpárrafo 1. 
integrante del ecosistema, equivalente a otras comunidades de seres vivientes. La Madre Tierra dispone de 7 derechos fundamentales: derecho a la vida, a la diversidad de la vida, al agua, al aire limpio, al equilibrio, a la restauración y a vivir libre de contaminación ${ }^{42}$. La naturaleza tiene derecho a mantener su integridad y a no sufrir alteraciones por las actividades humanas. La ley enfatiza el principio de bien colectivo: «El interés de la sociedad, en el marco de los derechos de la Madre Tierra, prevalece en toda actividad humana y por sobre cualquier derecho adquirido.» ${ }^{43}$ Asimismo, «el ejercicio de los derechos individuales está limitado por el ejercicio de los derechos colectivos en los sistemas de vida» ${ }^{44}$. El planeta y la comunidad viviente tienen prioridad absoluta ante cualquier interés particular.

El Estado se compromete a desarrollar tecnologías limpias, crear «...formas de producción y patrones de consumo equilibrados $»^{45}$ y defender y promover los derechos de la Madre Tierra y los principios correspondientes en el ámbito internacional. Se encargará de crear la institución especial, la Defensoría de la Madre Tierra ${ }^{46}$ para vigilar el cumplimiento de sus Derechos.

El reconocimiento legislativo de los derechos de la naturaleza y la creación de su estatus jurídico es sin duda un acto político excepcional y único en el contexto mundial. A la comunidad viviente en el planeta fue dada la misma importancia que tienen los seres humanos. La sociedad humana representa solamente una parte del ecosistema, con el que está indivisiblemente interrelacionada y con el cual comparte el destino común. En la Ley de Derechos de la Madre Tierra los postulados de la ecología profunda occidental (Lovelock 1987) ${ }^{47}$ resuenan con el concepto indígena de ayllu, en el que las dimensiones humanas y naturales son profundamente interdependientes (De Cadena 354).

Al igual que en la Nueva Constitución, en la Ley de Derechos de la Madre Tierra, a nivel teórico, la estrategia de protección del medio ambiente y el funcionamiento del Estado parecen complementarse en perfecta armonía. Pero en la realidad, muchos (si no la mayoría) de los artículos de la Ley son prácticamente negados por la política ejercida. El Estado boliviano se encuentra en una situación paradójica: si realmente quisiera cumplir con la Ley, debería redefinir completamente sus políticas económicas y-al fin y al cabo-reescribir capítulos enteros de la Constitución. Uno de los principios básicos de la Ley consiste en la

42. Capítulo III, Artículo 7, Párrafo I, Subpárrafos 1-7.

43. Capítulo I, Artículo 2, Subpárrafo 2.

44. Capítulo II, Artículo 6.

45. Capítulo IV, Artículo 8, Subpárrafo 2.

46. Capítulo IV, Artículo 10.

47. La hipótesis Gaia de James Lovelock considera la biosfera del planeta (todos los seres vivos junto con elementos anorgánicos) como un superorganismo con capacidad de autorregulación. En los años setenta, la hipótesis Gaia formó el marco teórico de la ecología profunda occidental. 
imposibilidad de mercantilizar los sistemas de vida ${ }^{48}$, que se definen como «... comunidades complejas y dinámicas de plantas, animales, microorganismos y otros seres y su entorno...bajo la influencia de factores climáticos, fisiográficos y geológicos».9 ${ }^{49}$ Pero, al mismo tiempo, la prioridad del Estado es «la industrialización y comercialización de los recursos naturales ${ }^{50}$. Según la lógica de la Ley, los recursos naturales, renovables o no renovables, forman parte indivisible de los sistemas de vida. Los ecosistemas, compuestos de organismos vivos y fenómenos anorgánicos, tienen derecho a mantener su integridad como una entidad viviente. La sociedad humana, como una parte del ecosistema, debería contribuir al mantenimiento de su equilibrio: consumir solamente lo que el ecosistema tenga capacidad de regenerar y generar residuos en la cantidad que el ecosistema sea capaz de absorber. En el marco de la Ley, el patrón actual de la explotación intensiva de los recursos no-renovables puede ser considerado como ilegítimo, violador de derechos fundamentales de la Madre Tierra y del principio de no mercantilización.

La política extractivista del Estado entra en conflicto con los derechos de la Madre tierra principalmente en el caso de la explotación de hidrocarburos. Otro ejemplo es la planeada industrialización del Salar de Uyuni, que fue proclamada la prioridad nacional en $2008^{51}$. Se estima que se encuentra en el salar más de la mitad de las reservas mundiales de litio $^{52}$. Para Bolivia, las ganancias sacadas de la comercialización del litio podrían ser enormes. Pero, según la Ley de Derechos de la Madre Tierra, el salar corresponde exactamente a la definición de un sistema de vida. Es una zona ecológicamente única con un ciclo anual de inundaciones regulares: en noviembre, la superficie inundada del salar y sobre todo el delta del Río Grande de Lípez se convierte en el lugar de cría de tres especies de flamencos. La biodiversidad del delta representa un fenómeno único a escala mundial (Hollender y Shultz 41). El frágil equilibrio ecológico del salar es sensible a cualquier intervención humana que lo puede alterar irreversiblemente. Del salar dependen, además, las comunidades locales: el agua es una fuente importante para los criadores de llamas, igualmente es usada en irrigación por los cultivadores de quinoa.

Para realizar el proyecto de la extracción del litio es necesario construir un sistema de piscinas de evaporación y salmueroductos que inevitablemente afectarán

48. Capítulo I, Artículo 2, Subpárrafo 5.

49. Capítulo II, Artículo 4.

50. Constitución Política del Estado, Cuarta Parte, Título II, Capítulo Segundo: Recursos Naturales, Artículo 355, Párrafo I.

51. «Acerca de la Gerencia», Gerencia Nacional de Recursos Evaporíticos (GNRE). Accesible en http:// www.evaporiticos.gob.bo/?page_id=124, consultado el 20 de enero de 2011.

52. "Salares de Bolivia», GNRE. Accesible en http://www.evaporiticosbolivia.org/index.php?Modulo=Te mas\&Opcion=SalaresBolivia, consultado el 5 de noviembre 2011 
el carácter del paisaje. Se producirá una gran cantidad de productos secundarios. Existe peligro de contaminación del río o aun de la desaparición completa de su agua a consecuencia del consumo industrial. No se conocen otros potenciales impactos ambientales de las futuras plantas de refinación. Brevemente, el proceso de la industrialización del litio probablemente tendrá un efecto devastador para el ecosistema del salar y para las comunidades locales (Hollender y Shultz 3947). Promoviendo este proyecto como prioridad nacional, Bolivia prácticamente viola los Derechos de la Madre Tierra y el principio de «vivir bien».

\section{¿Las dos Bolivias?}

Como tratamos de trazar en la reflexión precedente, Bolivia se encuentra en una situación paradójica y aun esquizoide. Intenta incluir los paradigmas indígenas en el concepto del Estado moderno y promueve el principio de «vivir bien». Dispone de un marco constitucional elaborado de la protección del medio ambiente. Al nivel internacional lucha por la justicia planetaria, critica las causas estructurales del cambio climático global y rechaza todas las formas del capitalismo. Pero al mismo tiempo practica lo que podemos llamar el capitalismo de Estado: basa su política económica en la extracción y comercialización de recursos naturales, a pesar de sus impactos negativos en los ecosistemas y comunidades indígenas. "Los petroleros son los soldados de la economía nacional.... ${ }^{53}$ afirma Evo Morales en contradicción con sus retóricas ambientales y promueve la intensificación de la explotación de hidrocarburos (Bebbington 2009: 14-18). Se preparan los megaproyectos de la industrialización del Salar de Uyuni o de la extracción del hierro en la mina gigante de Mutún ${ }^{54}$. A pesar de su postura anti neoliberal, Bolivia acepta la lógica del mercado libre y enfatizando el principio de la «competitividad ${ }^{55}$ comercializa sus recursos naturales en el mercado internacional. Los mayores ingresos los constituyen las ganancias de la industria hidrocarburífera ${ }^{56}$, los principales productos exportados son el gas natural y los minerales. Y el volumen de las exportaciones sigue creciendo ${ }^{57}$.

53. «Presidente Morales: Los petroleros son soldados de la economía nacional» Ministerio de hidrocarburos\&energía. Accesible en www.hidrocarburos.gob.bo/MHE2012/index.php?option=com_ content\&view=article\&id=1763:presidente-morales-los-petroleros-son-soldados-de-la-economianacional\&catid=108:noticias, consultado el 20 de enero de 2012.

54. «El proyecto de Mutún YPFB y Jindal», COMIBOL. Accesible en http://www.comibol.gob.bo/noticia_prensa/1282-El_proyecto_del_Mutun_YPFB_Y_Jindal, consultado el 20 de enero de 2012.

55. Constitución Política del Estado, Cuarta Parte, Título II, Capítulo Segundo: Recursos Naturales, Artículo 355, Párrafo III.

56. «Producto interno bruto por actividad económica», Instituto Nacional de Estadística. Accesible en http://www.ine.gob.bo/indice/general.aspx?codigo=40201, consultado el 26 de enero de 2012.

57. «Principales productos exportados al mundo según volumen y valon, IBCE: Instituto Boliviano de Comercio exterior. Accesible en http://www.ibce.org.bo/informacion-mercados/estad_bol/2010/diciembre/bolivia_exp_principales_100_prod_segun_vol_val.pdf, consultado el 25 de abril de 2012. 
Aunque el eslogan de «vivir bien» figura casi en todos los documentos gubernamentales ${ }^{58}$, en la realidad parece que el Estado boliviano sigue más bien el paradigma occidental de «vivir mejor».

Estas paradojas tienen sus causas estructurales profundas. Es indiscutible la necesidad de mejorar la situación socioeconómica de la mayoría de la población boliviana. El problema consiste en que el Estado boliviano asumió el concepto occidental del progreso y crecimiento económico basado en el aprovechamiento ilimitado de los recursos naturales. Debido a la dependencia respecto a la fluctuación de los precios de las materias primas en el mercado internacional Bolivia continúa envuelta en la economía global, que sigue la lógica occidental antropocéntrica según la cual la naturaleza es considerada tan sólo como una fuente de mercancía libremente explotable y negociable por los humanos (Bellamy Foster y Magdoff 8). Bolivia es socio o miembro asociado de varios tratados y proyectos internacionales (IIRSA, Mercosur, Comunidad Andina ${ }^{59}$, etc.), el Estado además figura como socio de las compañías trasnacionales ${ }^{60}$. El cumplimiento de estos compromisos es uno de los factores que de facto imposibilitan una verdadera reforma en las políticas económicas y la creación de un sistema alternativo, basado en los principios holísticos inspirados en las cosmovisiones indígenas.

Según nuestra opinión, una de las causas principales de la postura ambientalmente esquizoide del Estado boliviano consiste en su intento de armonizar dos conceptos inconciliables. Hay una discrepancia profunda entre los paradigmas indígenas y occidentales. A pesar de las reformas legales y la inclusión formal de ciertos principios indígenas en el marco constitucional y legislativo, en la práctica el Estado no abandonó la matriz política, económica y cultural occidental. Parece que realmente hay dos mundos incompatibles: el modelo occidental dominante de Estado y la visión indígena, comunitaria y profundamente interrelacionada con la Tierra (Quispe 1999). Aunque el gobierno de Evo Morales se legitimiza con el simbolismo indígena y a nivel teórico promueve derechos de la Pachamama, en la realidad continúa la política del Estado colonizador. Parece que son los mismos mecanismos sociales que caracterizó Quispe que imposibilitan un verdadero cambio hacia un sistema alternativo: el paradigma occidental simplemente dispone de más poder político, económico

58. «Plan Nacional de Desarrollo», Instituto Nacional de Estadística. Accesible en http://www.ine.gob. bo/pdf/PND/05.pdf, consultado el 24 de enero de 2012.

59. MERCOSUR. Accesible en http://www.mercosur.int/home.jsp?contentid=7\&seccion=1, IIRSA. Accesible en http://www.iirsa.org/index.asp?Codldioma=ESP, Comunidad Andina. Accesible en http://www. comunidadandina.org/, consultado el 10 de enero de 2012.

60. "Repsol descubre un nuevo yacimiento de gas en Bolivia», REPSOL. Accesible en http://www. repsol.com/es_es/corporacion/prensa/notas-de-prensa/ultimas-notas/09082010-repsol-descubre-yacimiento-gas-bolivia.aspx, consultado el 8 de enereo de 2012. 
y social. A nivel legislativo Bolivia hizo pasos únicos y el reconocimiento de estatus jurídico de la Madre Tierra (o el planeta) y sus derechos representan un reto para el resto del mundo. Pero no es posible promover dos conceptos esencialmente antagónicos. La participación en el mercado global y en las actividades de las compañías trasnacionales de facto niega el principio ético-moral de «vivir bien».

Es indudable que el proceso de modernización es necesario para asegurar las condiciones socioeconómicas para una vida digna de los bolivianos. Pero es un proceso que no es posible cumplir a corto plazo. El gobierno de Morales necesita mostrar los resultados positivos de su política en el marco de un periodo electoral. El extractivismo intenso es uno de los instrumentos que posibilitan conseguir relativamente rápido los recursos económicos necesarios para las reformas del país. Pero, al mismo tiempo es una postura que en la práctica contradice las bases ideológicas del proceso de cambio.

La única manera de salir de esta situación paradójica es empezar a planificar a más largo plazo. Hay que ir cambiando los patrones del consumo enfatizando en la producción y consumo a niveles locales. Esta estrategia podría contribuir al uso más efectivo del suelo que serviría prioritariamente para producir los alimentos básicos. Asimismo se podrían reducir las actividades explotadoras que, en la actualidad, están orientadas principalmente a la exportación. Produciendo y consumiendo a nivel local las comunidades podrían controlar directamente los impactos socio-ecológicos de sus actividades con el fin de mantener una relación equilibrada con su entorno. A más largo plazo, se podría alcanzar cierto nivel del autoabastecimiento alimentario y energético, que podría satisfacer de manera adecuada las necesidades de la población boliviana y cuyos excedentes podrían ser comercializados a nivel internacional. Esta estrategia puede apoyarse en el marco constitucional así como en la Ley de Derechos de la Madre Tierra.

Y finalmente, hay que asegurar la participación de los pueblos indígenas en el proceso de toma de decisiones políticas. Como demostró la protesta masiva en defensa del TIPNIS, ellos son los que realmente toman en serio el principio moral de «vivir bien» y la convivencia armoniosa con la Pachamama.

Agradecimiento: la autora agradece a la Fundación Científica Checa por el apoyo de su proyecto GAČR P404/10/P033, en el marco del cual fue escrita esta ponencia. 


\section{Bibliografía}

"Acerca de la Gerencia», Gerencia Nacional de Recursos Evaporíticos (GNRE). Accesible en http://www.evaporiticos.gob.bo/?page_id=124, consultado el 20 de enero de 2011.

«Acuerdo de los Pueblos», Conferencia Mundial de los Pueblos sobre el Cambio Climático y Derechos de la Madre Tierra. Accesible en http://cmpcc.org/acuerdo-delos-pueblos/, consultado el 20 de octubre 2011.

Bebbington, Antony. "The New Extraction: Rewriting the Political Ecology of the Andes?" NACLA Report on the Americas 42.5 (2009): 13-20.

Bebbington Antony \& Denise Humphreys Bebbington. "An Andean Avatar: Post-Neoliberal and Neoliberal Strategies for Securing the Unobtainable." New Political Economy 16.1 (2011): 131-145.

«Bolivia's President Urges Development of Economic System Based on 'How to Live Well'». United Nations: Permanent forum on Indigenous Issues. Accesible en http://www.un.org/News/Press/docs/2008/hr4944.doc.htm, consultado el 18 de abril 2012.

Borzutzky, Silvia \& Breanna Zwart. "Another Version of The Same Story: Is the 2009 Constitution Going to Make a Difference?” The Latin Americanist 53.4 (2009): $5-27$.

Calestani, Melania, "SUERTE (Luck): Spirituality and Well-Being in El Alto, Bolivia." Applied Research in Quality of Life 4:1 (2009): 47-75.

Chavez, Franz Bolivia, "Guarani, Tapiete Peoples Fight Gas Exploration." Global Information Network, Julio de 2010.

"Constitución Política del Estado », 2008, Ministerio de Culturas, accesible en http://www.minculturas.gob.bo/mdcb/normativa/cpe.pdf, consultado el 3 de agosto de 2011.

De la Cadena, Marisol, "Indigenous Cosmopolitics in the Andes: Conceptual Reflections beyond Politics." Cultural Anthropology 25.2 (2010): 334-370.

"El proyecto de Mutún YPFB y Jindal », COMIBOL. Accesible en http://www. comibol.gob.bo/noticia_prensa/1282-El_proyecto_del_Mutun_YPFB_y_Jindal, consultado el 20 de enero de 2012.

Foster, John Bellamy, \& Fred Magdoff. "What Every Environmentalist Needs to Know About Capitalism: a citizen's guide to capitalism and the environment." Monthly Review 61.10 (2010): 187.

GAVALDA, Marc. Las manchas del petróleo boliviano: tras los pasos de REPSOL en el territorio indígena Parque Nacional Isiboro Sécure, La Paz: FOBOMADE, 1999.

Healey, Susan. "Ethno-Ecological Identity and the Restructuring of Political Power in Bolivia." Latin American Perspectives 36.4 (2009): 83-100.

Hollender Rebecca, \& Jim Shultz. "Bolivia and its Lithium: Can the Gold of the $21^{\text {st }}$ Century Help Lift a Nation out of Poverty?” A Democracy Center Special 
Report, mayo de 2010, accesible en http://upsidedownworld.org/main/bolivia-archives-31/2507-bolivia-and-its-lithium-can-the-gold-of-the-21st-century-lift-anation-out-of-poverty, consultado el 22 de octubre de 2011.

Huanacuni Mamani, Fernando. Buen Vivir/Vivir Bien: Filosofía, políticas, estrategias y experiencias regionales andinas. Lima: CAOI (Coordinadora Andina de Organizaciones Indígenas), 2010.

Kimerling, Judith. "The 'Human Face of Petroleum': Sustainable Development in Amazonia?" Review of European Community \& International Environmental Law 10.1 (2001): 65-81.

« La entrevista con Evo Morales en la Conferencia Climática en Copenhagen el 17 de dicimebre del 2009 ", Democracy Now. Accesible en http://www.democracynow. org/2009/12/17/bolivian_president_evo_morales_on_climate, consultado el 15 de octubre 2011.

«Ley de derechos de la Madre Tierra», Gobernabilidad democrática en Bolivia, accesible en http://www.gobernabilidad.org.bo/noticias/2-noticias/704-bolivia-promulga-la-ley-de-derechos-de-la-madre-tierra, consultado el 9 de octubre de 2011. Lindisfarne, Nancy. "Cochabamba and Climate Anthropology." Anthropology Today 26.4 (2010): 1-3.

Lovelock, James. Gaia: a New Look at Life on Earth. New York: Oxford University Press, 1987.

"Plan Nacional de Desarrollo ", Instituto Nacional de Estadística. Accesible en http://www.ine.gob.bo/pdf/PND/05.pdf, consultado el 24 de enero de 2012.

"Por la defensa de la Madre Tierra, tierra y territorio ", CIDOB:Conferedación de los pueblos indígenas de Bolivia. Accesible en http://www.cidob-bo.org/index. php?option $=$ com_content\&view $=$ article\&id $=888$ :presidente-de-cidob-dio-conferencia-de-prensa-en-la-cop-17-en-durban\&catid=82:noticias\&Itemid=2, consultado el 17 de abril 2012.

«Presidente Morales: Los petroleros son soldados de la economía nacional» Ministerio de hidrocarburoserenergía. Accesible en www.hidrocarburos.gob.bo/MHE2012/ index.php?option $=$ com_content\&view $=$ article\&id $=1763$ :presidente-morales-lospetroleros-son-soldados-de-la-economia-nacional\&catid=108:noticias, consultado el 20 de enero de 2012.

«Producto interno bruto por actividad económica ", Instituto Nacional de Estadistica. Accesible en http://www.ine.gob.bo/indice/general.aspx?codigo=40201, consultado el 26 de enero de 2012.

Quispe Huanca, Felipe. El indio en escena. La Paz: Ediciones Pachakuti, 1999.

Ramirez, Susan. To feed and Be Fed: the Cosmological Bases of Authority and Identity in the Andes. Stanford: Stanford University Press, 2005.

"Repsol descubre un nuevo yacimiento de gas en Bolivia ", REPSOL. Accesible en http://www.repsol.com/es_es/corporacion/prensa/notas-de-prensa/ 
ultimas-notas/09082010-repsol-descubre-yacimiento-gas-bolivia.aspx, consultado el 8 de enereo de 2012.

«Resolución Aprobada por la Asamblea General, 63/278. Día Internacional de la Madre Tierra ", Naciones Unidas. Accesible en http://www.un.org/es/comun/ docs/?symbol=A/RES/63/278, consultado el 20 de octubre 2011.

"Salares de Bolivia », GNRE. Accesible en http://www.evaporiticosbolivia.org/index.php?Modulo=Temas\&Opcion=SalaresBolivia, consultado el 5 de noviembre 2011.

Resumen: Bolivia es el país con mayor porcentaje de la población indígena en Sudamérica. Es también uno de los primeros países donde fue ratificada la ley de Derechos de la Madre Tierra. Inspirándose en las cosmovisiones aymaras y quechuas, Bolivia extendió el concepto de derechos humanos a la naturaleza, que se volvió una entidad equivalente a la sociedad humana. Pero al mismo tiempo, el gobierno boliviano empezó el proceso de modernización y reformas sociales financiadas primeramente por las ganancias de la comercialización de los recursos naturales, especialmente hidrocarburos. Uno de los planes estratégicos de Bolivia es la industrialización del litio en el Salar de Uyuni, una región ecológicamente única. Es este curso económico y la dependencia del mercado global compatible con el principio indígena de «vivir bien»? «Tratamos de vivir bien, no vivir mejor. Vivir mejor significa vivir a costa del otro.» ¿Son estas palabras de Evo Morales válidas en el marco de la orientación política actual de Bolivia?

Palabras claves: Bolivia, Derechos de la Madre Tierra, Constitución Política del Estado, hidrocarburos, litio

Résumé : La Bolivie est le pays qui présente le pourcentage le plus élevé de population indigène en Amérique du sud. C'est aussi l'un des premiers pays à avoir ratifié la loi sur les Droits de la Terre Mère. S'inspirant des cosmovisions aymara et quechua, la Bolivie a étendu le concept de droits humains à la nature, laquelle est devenue une entité équivalente à la société humaine. Mais dans le même temps, le gouvernement bolivien a entrepris un processus de modernisation et de réformes sociales financées en premier lieu par les profits tirés de la commercialisation des ressources naturelles, en particulier des hydrocarbures. L'un des plans stratégiques de la Bolivie est l'industrialisation de l'exploitation du lithium des marais salants de Uyimi, une région unique au plan écologique. Cette évolution économique et la dépendance vis-à-vis du marché mondial sont-elles compatibles avec le principe indigène du « bien vivre » ? « Nous essayons de bien vivre, et non de vivre mieux. Vivre mieux signifie vivre aux dépens des autres ». Ces propos de Evo Morales ont-ils une réelle portée au vu de l'orientation politique actuelle de la Bolivie?

Mots-clés : Bolivie, droits de la Terre Mère, constitution politique de l'Etat, hydrocarbures, lithium 